\title{
Implementation of an Experimental Wide-Area Monitoring Platform for Development of Synchronized Phasor Measurement Applications
}

\author{
Moustafa Chenine, Member, IEEE, Luigi Vanfretti, Member, IEEE, Sebastian Bengtsson, and \\ Lars Nordström, Member, IEEE
}

\begin{abstract}
Synchrophasor-based Wide-Area Monitoring and Control Systems (WAMC) are becoming a reality with increased international research and development. Several monitoring and control applications based on these systems have been proposed, and although with a relative small adoption, they are currently supporting the operations of some large transmission system operators. It is expected that the continued research and development of phasor data applications will enable the miracle of "Smart Grids" at the transmission level. The authors have realized that this can be achieved in timely fashion only if a research and development platform is developed to simultaneously address issues regarding information and communication infrastructures, and phasor data applications. This paper discusses the preliminary development, and deployment of an experimental wide-area monitoring and control platform in which several basic applications have been implemented, and that in the future will allow for the implementation and testing of envisioned applications. At its current stage, the platform allows both online monitoring and off-line analysis. In the future, it will be the cornerstone to a wider platform enabling research on phasor data applications that intrinsically account for ICT aspects.
\end{abstract}

Index Terms-Wide Area Monitoring System, Wide Area Monitoring and Control Systems Performance, PMU-based Applications.

\section{INTRODUCTION}

$\mathbf{T}$ HE rising interest in "Smart Grids" has prompted an everincreasing wave of discussion regarding a more disruptive introduction of information and communication technologies (ICT) to increase efficiency in electricity delivery and power network management. While a great amount of discussion focuses on the increased use of ICT at the distribution level [1], synchronized phasor measurements and their supporting infrastructures are put in the front-line from the transmission system perspective as enablers of the so called "Smart Transmission Grids" [2].

The current approach for power system operations at the transmission level is to perform most of the monitoring and

Invited Paper, International Implementation Experience and Prospective Applications of Synchrophasors and their Supporting Infrastructures Panel Session, IEEE PES GM 2011.

This work was supported in part by the Swedish Power industry via ELEKTRA project 36084, and EKC ${ }^{2}$ - The Swedish Centre of Excellence in Electric Power Engineering.

M. Chenine and L. Nordström are with the Industrial Information \& Control Systems Division, School of Electrical Engineering, Royal Institute of Technology (KTH), SE-100 44, Stockholm, Sweden. E-mail: moustafac@ics.kth.se, larsn@ics.kth.se

L. Vanfretti and S. Bengtsson are with the Electric Power Systems Division, School of Electrical Engineering, Royal Institute of Technology (KTH) Teknikringen 33, SE-100 44, Stockholm, Sweden. E-mail: luigiv@kth.se. Luigi Vanfretti is supported by the STandUP for Energy collaboration initiative and the KTH School of Electrical Engineering.

978-1-4577-1002-5/11/\$26.00 C2011 IEEE control actions within an Energy Management System (EMS), which makes use of a Supervisory Control and Data Acquisition (SCADA) system. There are many solutions available and currently used by transmission system companies and system operators, such as ABB's SCADA/EMS Network Manager [3]. Although these systems are mature and dependable, it has not been until recently that wide-area "features" have been added to these systems. However, these features are not broadly adopted and have a reduced number of available phasor-data applications [4]. In addition, these systems where not developed to withstand the strain of managing the data volumes from the streaming of synchronized phasor measurements in an efficient manner [5]. Despite these limitations, there are initiatives in North America which have created specialized systems exploiting measurements from Phasor Measurement Units (PMUs) with the aim of enabling new applications of PMU-data and increasing the utilization of synchrophasors in operations [6].

The enabling technologies of wide-area monitoring systems are PMUs, as the measurement device of choice, and their supporting infrastructure which is formed by communication networks and computer systems capable of handling PMU data and other information (usually called Phasor Data Concentrators (PDCs)). The set of PMUs and their enabling information and communication infrastructures is termed Synchrophasor Measurement Technology (SMT) [7]. With the rising number of synchrophasor installations around the world, stakeholders in the transmission system have a window of opportunity to exploit synchronization, dynamic signatures, and high resolution measurements provided by PMUs. However, the number of applications available to transmission operators for exploiting these measurements seems not to be sufficient to justify investments in SMT, and current projects dealing with this technology are strongly subsidized [8].

If future power systems will hinge on these technologies, they cannot rely on heavily subsided funding as its means to existence, and other drivers are needed. These drivers can be provided by the careful design of reliable phasor data applications that provide an added value for operations and planning, a value which cannot be achieved by other technologies. But to make this a reality, both pieces of the puzzle must be addressed simultaneously: this means that the chosen Research and Development $(\mathrm{R} \& \mathrm{D})$ approach should simultaneously address issues regarding information and communication infrastructures, and phasor data applications. Hence, the approach proposed in this article is to develop an application development platform that, because it's based on open source software solutions, it 
respects the freedom of the researchers from both ICT and power engineering to develop phasor data applications.

\section{A. Purpose}

In this paper we present preliminary work that has focused on developing a Wide-Area Monitoring System and basic applications as part of a wider platform for "Smart Transmission Grids" application development and testing at KTH, the Royal Institute of Technology in Stockholm, Sweden.

\section{B. Outline}

The reminder of this paper is structured as follows. Section II presents an overview of the basic components of a WAMC system. Section III presents the architecture of the Wide Area Monitoring system under development and discusses its main components, while Section IV describes efforts to develop on-line and off-line applications. Finally, in Section $\mathrm{V}$, conclusions are drawn and future work is briefly outlined.

\section{BASIC WAMC COMPONENTS}

In the current centralized architecture paradigm, a complete Wide-Area Monitoring and Control (WAMC) System is one where PMU measurements are collected from various locations in an interconnected power system. These measurements are then communicated to a central location where they are used by an assessment or monitoring application that raises alarms and calculates metrics. The alarms raised and computed metrics by these monitoring systems are in turn used to provide operator support to determine corrective actions and to control devices in the power network. Alternative architectures, such as using a few remote PMU signals in a local system for specific control or protection applications, are also possible, but are outside the scope of this article.

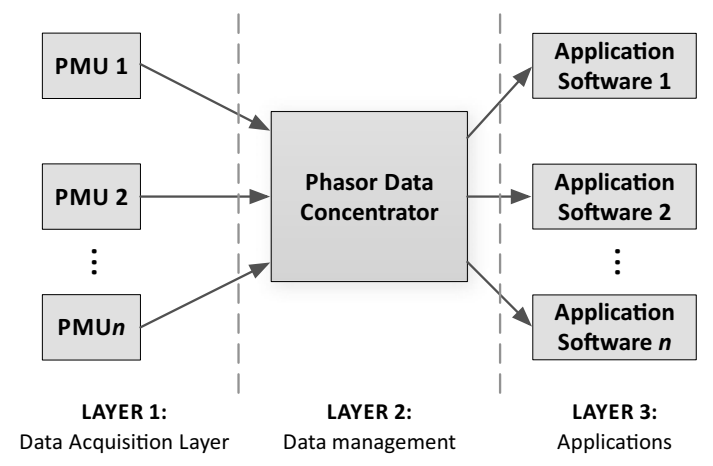

Fig. 1. Layers and components of a basic WAMC systems

Thus, the basic components of any WAMC system are the following: PMUs, PDCs, a PMU-based application system (which itself is comprised by different information technologies and underlying algorithms), and finally a communication network [9].

Similarly to traditional SCADA systems, there are three layers in a WAMC system. Figure 1 illustrates this logical architecture. In Layer 1, the WAMC system interfaces with the power system on substation bars and power lines where the PMUs are placed, this is called the Data Acquisition layer. Layer 2 is known as the Data Management layer, in this layer the syncrhophasor measurements are collected and sorted into a single time synchronized dataset. Finally, Layer 3 is the Application Layer; it represents the real-time PMU data-based application functions that process the time-synchronized PMU measurements provided by Layer 2 .

\section{Architectural Design of AN OPEn WAMS PLATFORM}

The openWAMS platform currently deployed at KTH is comprised by several components. These include components that sort PMU data and those that exploit the sorted data. The PMUs are deployed in various universities in the Nordic Region, and also include an FNET device. This section provides an overview of openWAMS, beginning with the overall architecture of the system and then describing the main parts that compose it. Most of these components have been deployed or are undergoing development, while others are still under planning.

\section{A. Overall Architecture}

Figure 2 depicts the main components of the Wide-Area Monitoring platform. As mentioned earlier, PMUs are deployed in various Universities in the Nordic region. Most of these PMUs are actually connected to the distribution grid, rather than the transmission grid. The PMUs have been equipped with step-down transformers that bring the threephase distribution level voltage to instrumentation level; the current is not being measured by these instruments. There are currently 5 PMUs deployed of which 4 are operational. Furthermore, a Frequency Disturbance Recorder (FDR) device [10] is also deployed at KTH which streams data both to the openWAMS platform, and the F-NET server at University of Tennessee [11].

There are currently plans to integrate a real-time power system simulator to the system, whereby measurements, device's

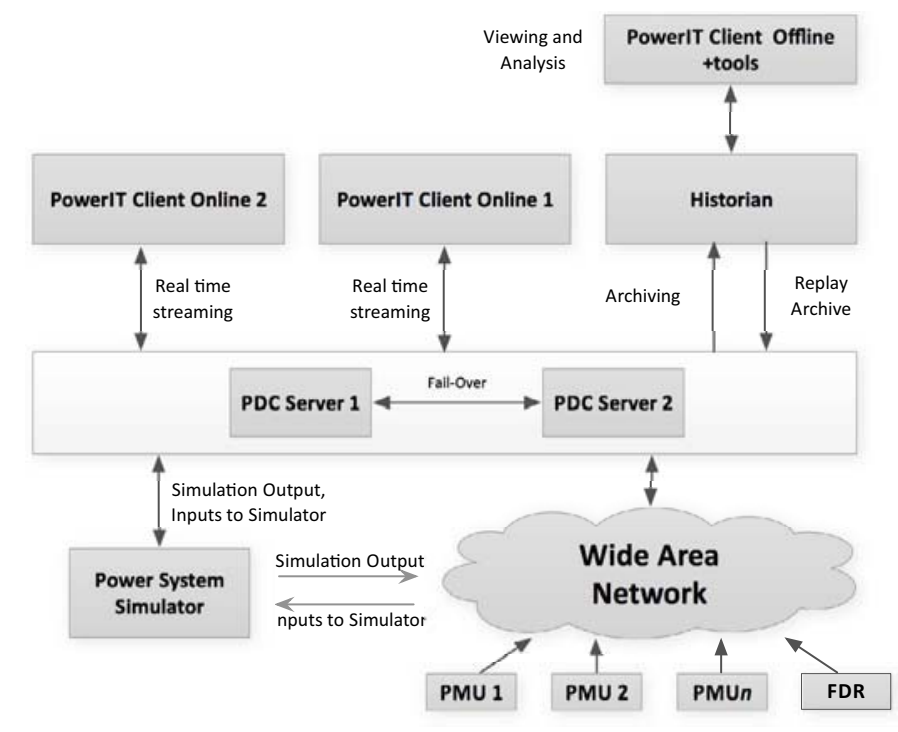

Fig. 2. The KTH openWAMS wide-area monitoring platform 
status, and other data can be extracted from the simulator and be feed into the WAMS system. This is attractive because by exploiting the input/output capabilities of the simulator, it becomes possible to implement and test closed-loop applications.

There are currently two PDCs deployed, these PDCs collect measurements and forward them as a single continuous data stream to either the historian system (which archives the data) or to client systems (which consume the data). In the case of the latter, the data stream from the PDC is time-aligned and may be down-sampled.

\section{B. openPDC}

The PowerIT Platform is built around the openPDC system [12]. openPDC is an evolution of the SuperPDC originally developed at the Tennessee Valley Authority [13]. The system processes any type of time-series measurement data in a protocol independent fashion. This is possible due to the system's extensible modular design. In fact, openPDC is architecturally split into three layers, the Input, Action and Output layers, each with its specific set of functionality, Fig. 3 illustrates this architecture. The Input, Action and Output layers deal with measurement acquisition, measurement processing and measurement routing and forwarding tasks respectively.

The Input Adapter Layer allows the development of input adapters that can support several protocols. An input adapter takes measurements from different devices or systems that use different communication protocols and makes them available to the Action Layer. Currently openPDC supports input from devices using IEEE C37.118, IEEE 1344, Virginia Tech FNET, and BPA PDCStream, among others [13]. The input adapter also allows development of customized inputs for interfacing with other systems. For example, openPDC supports reading archived data from a historian (as explained in the next section) back into openPDC. Other customized inputs can be developed to link conventional SCADA systems or interfaces sending simulated outputs from power system simulators/emulators.

The Action Adapter Layer, allows the implementation of functionality that process the stripped down measurements provided by the Input Adapter Layer. A good example of such functionality is the phasor time alignment adapter, where

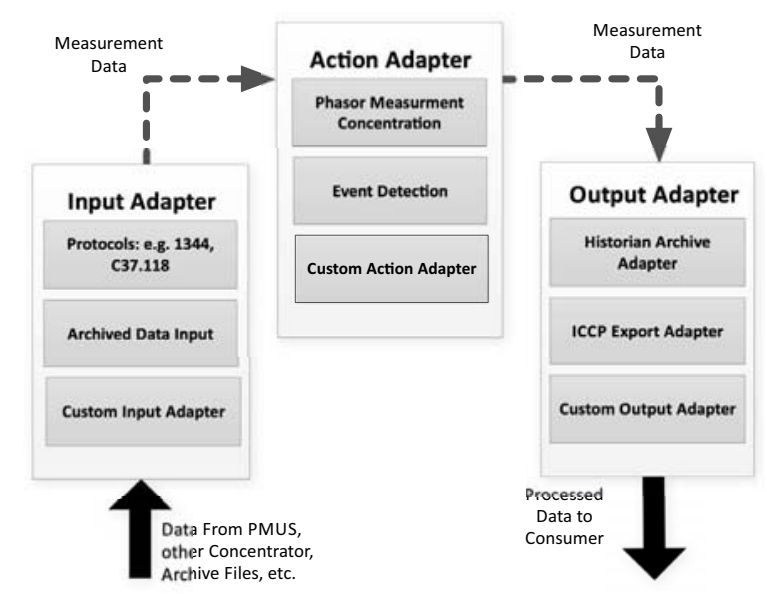

Fig. 3. Functionality Layers in openPDC's architecture measurements coming from the Input Adapter Layer are sorted, time-aligned and possibly down-sampled before being forwarded to the next layer. The Action Adapter Layer also supports two more functionalities which are of interest, namely real-time event detection and real-time calculated measurements. Real-time event detection tasks, process incoming measurements and checks whether they fall within a range of pre-set parameters, if they don't than the event task informs subscribers of such occurrences, an example of an event detection task that is bundled with openPDC is frequency excursion detections [14]. The real-time calculated measurement functionality allows for the definition of new measurements that are not directly collected from remote devices, but are instead calculated at the PDC from remote measurements as they arrive. A simple example of such a calculated measurement that can be derived is active power, which would be calculated within openPDC and then forwarded to applications requiring it, or to other adapters.

Finally, the Output Adapter Layer of the openPDC provides functionality whereby measurements processed by Action Adapter Layer are queued for processing or forwarded to subscribers. This includes the possibility of re-encapsulating the data in several protocols. Examples of output adapter bundled with openPDC are IEEE C37.118 concentrator output streams, Inter Control Center Protocol (ICCP) file export, Comma Separated Values (CSV) File Export, and Historian Archiving output.

The openPDC system deployed for the openWAMs currently uses IEEE 1344 to communicate with most PMUs and IEEE C37.118 to communicate with one PMU. Furthermore the FDR deployed at KTH uses F-NET to communicate with the PDC. In all cases the concentrated output stream is defined in IEEE C37.118.

As mentioned earlier, there are two openPDC system deployed as part of the openWAMS, this is done to study builtin load balancing and fail-over capabilities. In the former the back-up PDC assists the primary PDC when the traffic load increases, so as to maintain performance. In the latter the backup PDC is bought on-line when the primary PDC fails. These features are somewhat possible to configure with open PDC and our deployment is meant to study these configurations and performance in such cases.

Another interesting feature is data replay using the openPDC. In this case, archived data from the Historian (see next subsection) is retransmitted to the openPDC software which in turn treats the data as if it is coming from PMUs and re-streams the data back to the client applications, in this case the PowerIT client system.

\section{Historian}

The historian system is built around the openHistorian [15]. The openHistorian system is very much related to the openPDC system, in fact they are built using the same architectural framework, and is as extensible as the openPDC system.

The main function of the Historian is to archive and store measurement data for future off-line analysis use. The Historian stores the data in flat binary archive files. It does not use a 


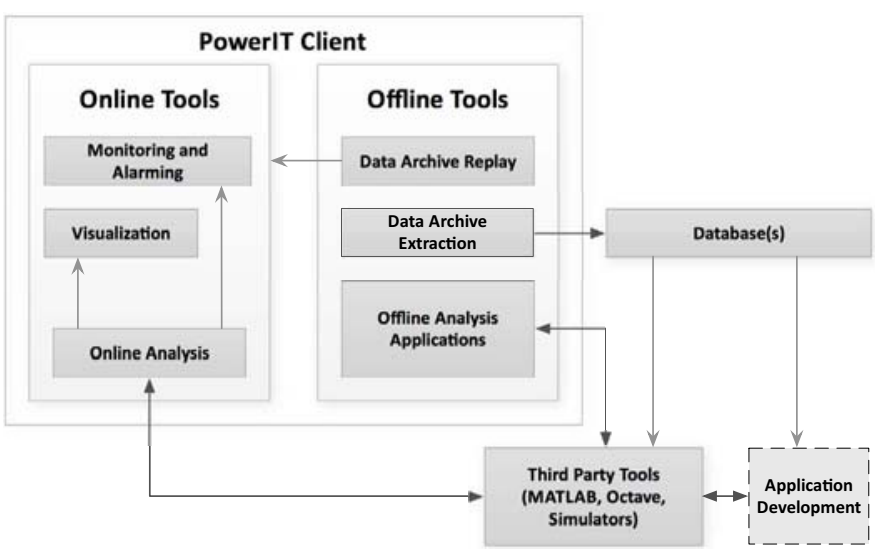

Fig. 4. Functionality of the PowerIT Client System

conventional relational database management system as such systems become inefficient when managing large amounts of data. This is because they would require large indexes and it would take a substantial amount of time to retrieve the required data [16].

As depicted in Fig. 2, the Historian is a standalone server system that receives a stream of data from the PDC and stores that data locally, that data storage facility is currently 2 terabytes but that can easily be extended increase storage capacity.

\section{PowerIT Client system}

The purpose of the PowerIT client system is to offer a platform for on-line and off-line analysis, and testing. Fig. 4 below gives an overview of how functionality is partitioned in the PowerIT client and associated tools. The system has the following application categories:

1) On-line real-time monitoring.

2) On-line real-time event processing.

3) Off-line replay of data for monitoring.

4) Off-line replay of data for analysis.

5) Server and client management.

This system has been designed to offer a multiuser environment where users have access to certain system functionality according to assigned rights, such as on-line monitoring, offline analysis tools, and server configuration.

The PowerIT client system makes use of many of the libraries associated with the openPDC, for example the TVA Code Library [17] and openPDC's Phasor Protocol Library.

The architecture of the PowerIT client is fairly straightforward. The system is built around a reconfigurable connection object that can connect using several protocols to either a PDC server, measurement historian and or a remote device.

The applications in the system are designed to plug-in to the global connection object and subscribe to events fired by that object. A global connection object event can be for example a new measurement from the server that has been received, in which case, the applications responds by utilizing this measurement according to its task.

Applications also import a global configuration manager. The global configuration manager simplifies the management of various configurations related to connection between the

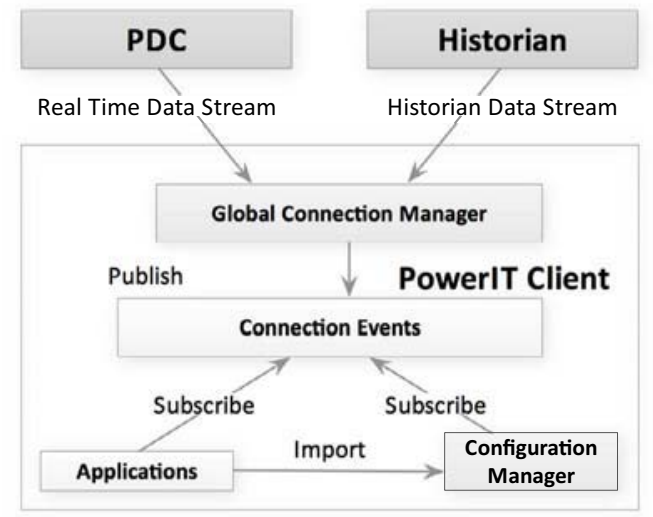

Fig. 5. The main architectural component of the PowerIT client system

client system and the PDC. The configuration manager can also handle configuration changes in remote devices if the client is directly connected to them (and therefore bypassing the PDC). An example of the kind of information handled by the configuration manager is the number of measurements published by the PDC server and what type of measurements they are, i.e. voltage phasors, frequency values etc. In fact, the configuration manager also subscribes to the events of the global connection object but only to those events relating to configuration changes. By importing the configurations manager, application can dynamically respond to changes in the system configuration. Figure 5 depicts the main architectural components of the PowerIT client system.

As illustrated in Fig. 4, the PowerIT client systems is also designed to offer tools to support off-line analysis and data management such as tools to easily extract archive data for viewing directly in the application or for processing inside other application systems like MATLAB.

\section{Application DeVelopment USing an open WAMS PLATFORM}

Using the infrastructure and components described in the previous section, several applications where built. These applications as mentioned earlier, are generally categorized as online or off-line applications. On-line application process fast moving data as they arrive to the client system, measurement by measurement. In contrast off-line applications process data that is archived and stored, and this is usually done on a batch volume of data.

\section{A. Online Monitoring Applications}

Two on-line applications have been implemented in the PowerIT client system. The first application is an average frequency monitoring display, and the second application is an online electromechanical model estimation system. The next two subsection describe these applications

1) A Simple Average Frequency Monitoring Application: The Average Frequency Monitoring application in the PowerIT client calculates the average frequency given a set of frequency values from several PMUs. The application is an adaption of the Frequency Monitoring function of the Power Calculations Adapter that is bundled with openPDC [14]. 


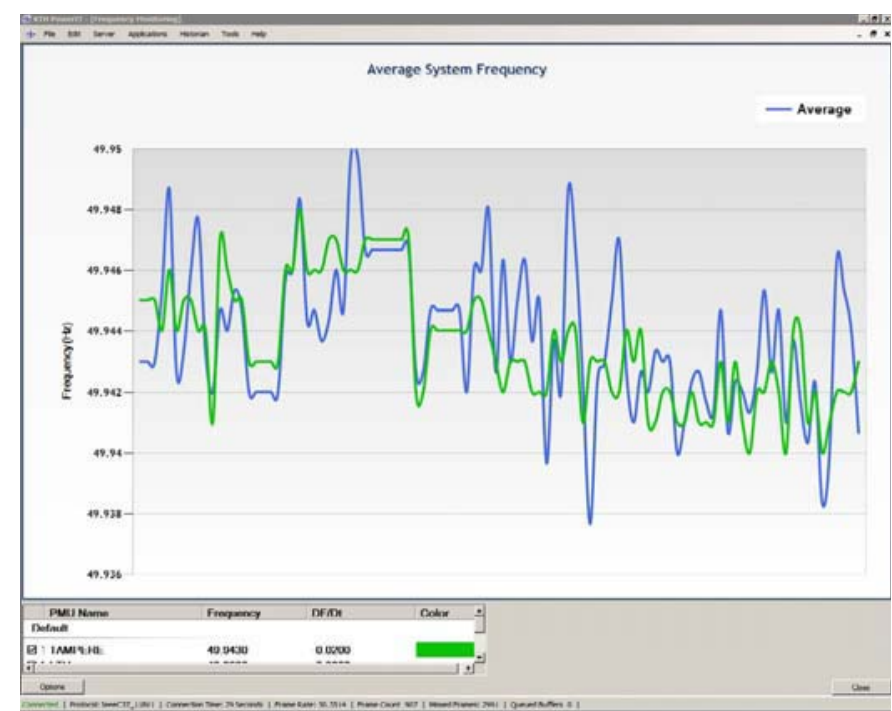

Fig. 6. Average Frequency Display - The blue line shows the computed average frequency from all the devices, the green line shows the frequency from the TAMPERE PMU, the $x$-axis is a moving window of configurable size.

The Average Frequency Monitoring application calculates the average frequency as each value arrives at the client system this is done simply by adding each frequency value to the a "Frequency Total" variable and then dividing by the number of frequency values received.

The application also allows plotting the frequency of each PMU on the same graph as the average frequency trend. Figure 6 shows the average frequency calculated from three PMUs as the blue curve while the green curve represents the frequency of a PMU in the south of Finland (named TAMPERE).

2) On-line Electromechanical Mode Estimation: The online mode estimation application is based on work outlined in [18] where archived data is used to estimate electromechanical properties of power systems. The application collects realtime measurements for a pre-specified time window and then processes these measurements in two steps: first preprocessing the data buffer, and then performing a spectral estimation.

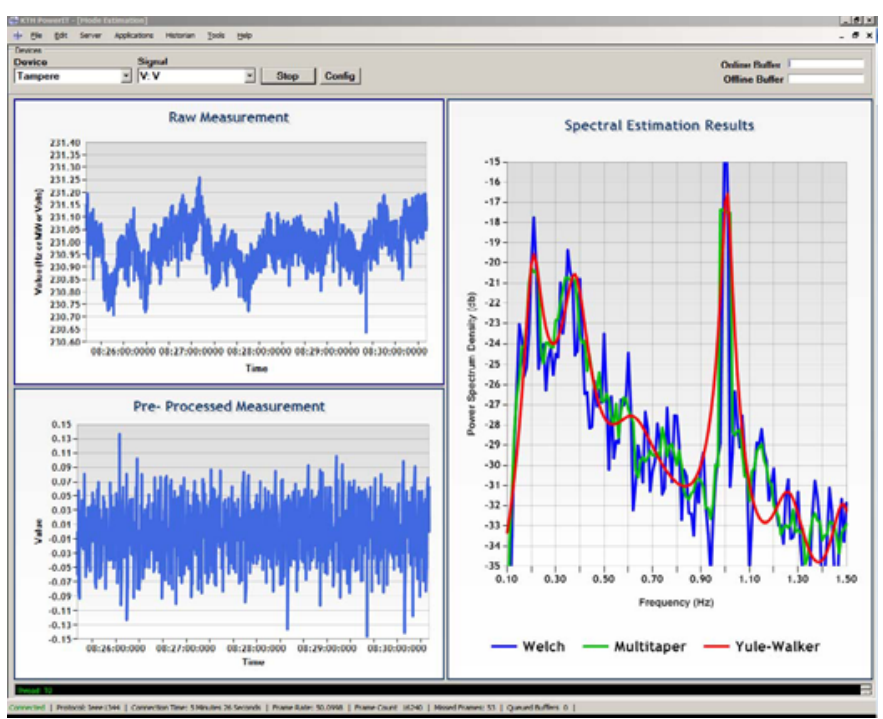

Fig. 7. The on-line Mode Estimation display in the PowerIT client system

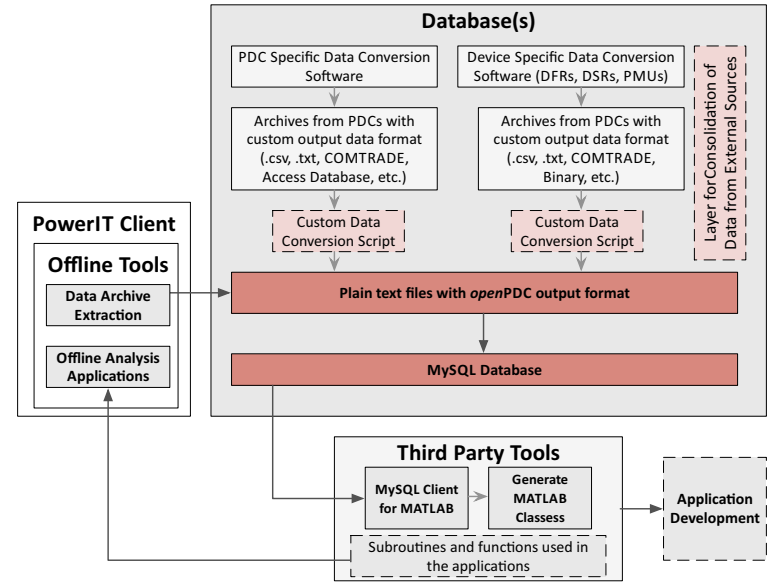

Fig. 8. Data consolidation layer and data management approach for off-line analysis application development

Figure 7 below shows a screen shot of this application.

The processing part of the mode estimation application was originally written in MATLAB. This code was reorganized in to more manageable functions and exported using the MATLAB NE Compiler [19] as independent libraries to be used by the mode estimation application component of the PowerIT client system.

\section{B. Off-line Analysis Applications}

Off-line analysis can be generally categorized in two areas: forensic or post-mortem disturbance analysis, and application development. Availability of data for forensic analysis is of importance because of regulations [20] and it often allows the determination of factors involved in disruptive events [21], [22]. Application development refers to the use of archived data for developing new analysis applications that can be used either in on-line and off-line mode. We discuss some of these aspects in this Section.

1) Data Consolidation Layer and Data Management Approach: Despite that the COMTRADE format has been selected for PMU data sharing in North America [23] this data format is not the most convenient for application development and straightforward data analysis. Moreover, the PMU data may not necessarily come from PDCs other than the one owned by the analyst, and may bear a different output data format than the one provided by openPDC. In addition, for analysis purposes it might be necessary to consolidate data from other GPS-time synchronized measurement devices (such as Disturbance Frequency Recorders (DFRs) and Dynamic Swing Recorders (DSRs), and other IEDs [24].

PMU data is available from different sources: PMUs, IEDs, and PDCs. In addition to the different sources, the data is also exchanged in many different formats: COMTRADE [25] .cfg and dat files, delimited text files such as .csv and MS Excel files, and database files from MS Access and MySQL. To handle the diversity of data sources and data formats, and to cater for both the needs of the data analyst and application developer, we propose a data management approach which uses a layer for consolidation of data from different sources.

The data management approach consists of consolidating different data sources as shown in Fig. 8. The most important 
characteristic to note here is that the PowerIT Client provides a "Data Extraction" adaptor. This adaptor can be configured to provide data in different formats. However, because the output data format that is mainly used routinely is the one of open $\mathrm{PDC}$, the main goal is to transform the different data sources into this format.

When the data from an external PDC has been extracted to a text delimited format or other by the manufacturer's software, a custom conversion script changes the format of the data to the openPDC format, and stores it in plain text or .csv files. A similar approach is taken for consolidating data from specific devices that are not connected to a PDC such as DSRs and DFRs.

Once all the data is available in .txt or .csv files in the same format as data extracted with the "Data Extraction" adaptor, the data bears following format:

$\{0: I\} ;\{1: T\} ;\{2: V\} ;\{3: Q\}$

where $I$ is an internal historian identifier for the signal, $T$ is a time-stamp, $V$ is the value of the signal at the instant given by $T$, and $Q$ is a data quality indicator. In this case the delimiter ";" separates the identifier, time stamp and value.

For efficient data handling a relational database management system, MySQL, creates a database from the .csv files. The MySQL Client for MATLAB [26] enables this process from within the MATLAB environment, and also imports data from the MySQL database to MATLAB.

When a MySQL database is created the data is loaded into a table. The table contains the columns ID, Time Tag, Value and Quality. Data can then be taken from the table, by selecting an ID, to create .mat files. One of the great benefits of doing this is that the data that comes partially unsorted from openPDC now can be sorted by the ascending order of the time-stamp in MySQL. This is done by implementing the proper SQL query within the MySQL client.

All of these steps are not done manually, a small Graphical user interface (GUI) was constructed in MATLAB's GUI Design Environment (GUIDE). It incorporates the conversion process from the point where the data is in common format all the way to MATLAB, Fig. 9 below illustrates this GUI.

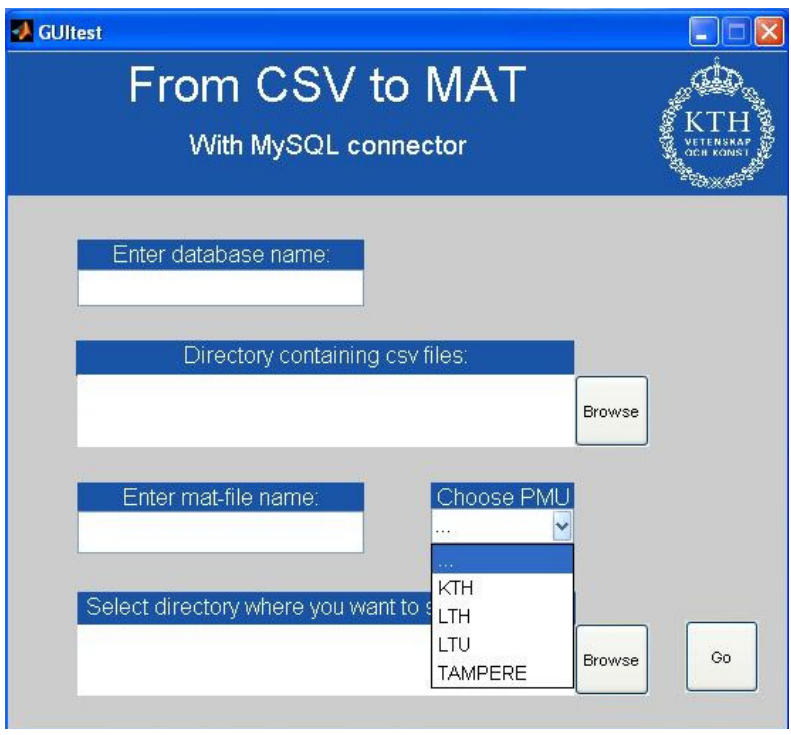

Fig. 9. MySQL to MATLAB data conversion GUI

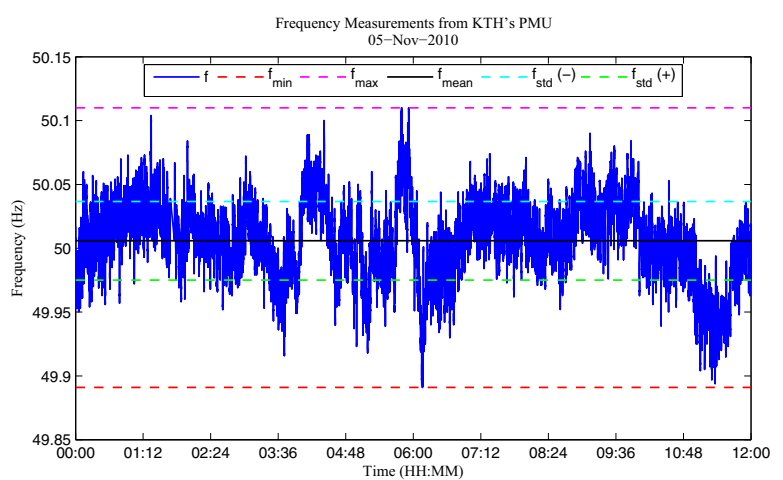

Fig. 10. Frequency measurement analysis off-line application

2) PMU Data Attributes for Data Analysis and Application Development: MATLAB data is organized in classes and structures for particular dates or events. Data structures and classes provide a compact and organized mean to handle PMU data for analysis: each signal from a PMU is defined as a class or structure, and each of the attributes of this signal is defined as a field. To illustrate these features, some of the fields of the structure for the frequency signal of the PMU at KTH $\left(k t h \_f\right)$ are shown below:

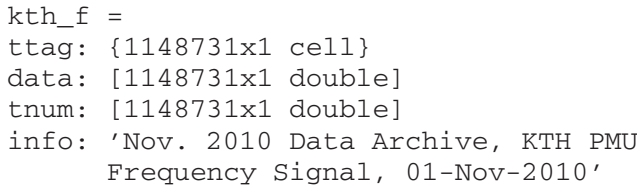

Note that a given class can bear additional data structures or fields providing other information for analysis and software development, as shown by the $k t h \_f$. info field. Using data structures allows the inclusion of not only the data, but also additional information about the signal.

3) Development of off-line applications: Data analysis can be readily carried out by having all the data stored in data structures. Operations, manipulation, and visualization can be standardized and done quickly.

a) Frequency measurement monitoring application: A simple off-line application for extracting features of frequency measurements from the PMU at KTH was recently developed; this example helps to show why the chosen data management approach is important. This application consists of a set of MATLAB functions that provide plots of measurement data for any time interval, and that computes different statistics on the data which are displayed in the plot and are provided also as an output of the application. A plot from the output of this application is shown in Fig. 10, within this application the statistics are computed by using the signals attributes:

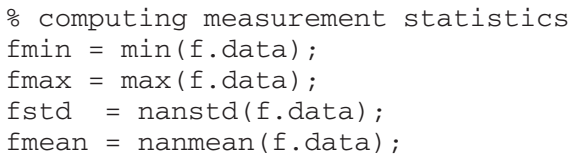

Note that the kth_f structure is received by the application in the new structure " $\mathrm{f}$ ", this generalization allows the application to be used by data from any other PMU. 
There are several applications currently being developed using this approach. Note that as explained above, the availability of data in a transparent and ready to use format allows for development to be pursued in a straightforward manner.

b) Electromechanical mode estimation application: The on-line mode estimation described in the previous Section has its roots in the on-going development of an electromechanical mode estimation off-line application. This application is being developed so that electromechanical mode properties can be routinely estimated from archived data from different power systems [18]. The application estimates the frequency spectrum of a given signal using different spectral techniques, and computes damping estimates using energy methods [27]. The application is built using three steps, the first step being preprocessing of data, the second is spectral estimation, and the third is damping estimation. The application has been designed so that any spectral technique can be evaluated and studied by including it in a separate function; these techniques include parametric and non-parametric methods. Discussion of these techniques is out of the scope of this paper and can be found in [28]. A sample output of this application from spectral estimation is shown in Fig. 11 for a data set from the Mexican Power System, a GUI similar to the one in Fig. 9 is currently being developed.

\section{CONCLUSions AND FurTher WORK}

In this paper we presented preliminary work on the development of an open Wide Area Monitoring system. The openWAMs platform is made up several components, most of which are open source. This has allowed us to experiment with different architectures and to begin building new monitoring

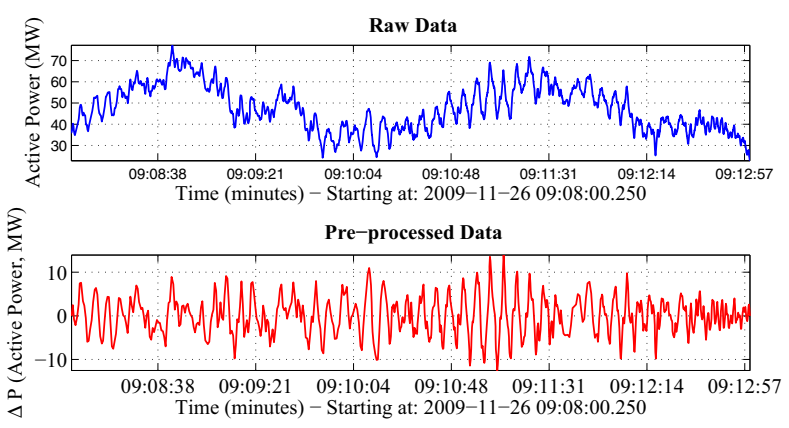

(a) Graphic output from data pre-processing

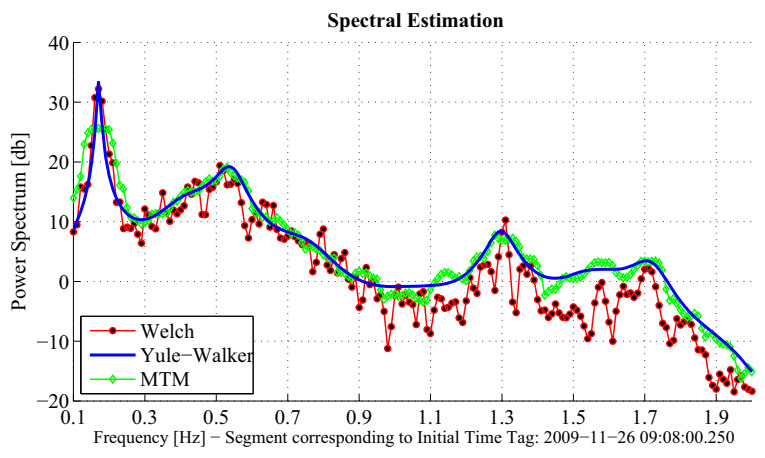

(b) Graphic output from spectral estimation

Fig. 11. Graphic outputs from the mode estimation application and analysis applications, some of which were presented in this paper. The system is a valuable asset as it helps expedite research and development efforts within the field of wide area situational awareness in terms of algorithms implemented in practice using real data, which would otherwise be solely based entirely on simulation results. The platform also helps in determining and evaluating the impact and limitation of ICT systems on the genuine reliability and usability of these algorithms, again this is done by implementing algorithms and evaluating their performance on real data in real-time fashion as opposed to off-line batch processing.

On-line applications that process sub-second incoming continuous streams of measurement data have a greater number of challenges and constraints. These applications have to check in real-time the quality of the data before processing it. If the data was inaccurate or distorted this could lead to an application failure or worst, producing misleading results which could deceive the operators. Another aspect is the overwhelming volume of incoming data that a client system has to process, which could inhibit performance. Future work will focus on implementing more algorithms and evaluating such ICT challenges and constraints.

\section{ACKNOWLEDGEMENTS}

The authors would like to express their gratitude to $\mathrm{J}$. Ritchie Carroll, Paul Trachian and the rest of the openPDC development team for their efforts and time in providing a quality and timely open source software solution which has given us the freedom to experiment.

\section{REFERENCES}

[1] G. Heydt, "The Next Generation of Power Distribution Systems," IEEE Transactions on Smart Grid, vol. 1, no. 3, pp. 225 -235, Dec 2010.

[2] A. Bose, "Smart Transmission Grid Applications and Their Supporting Infrastructure," IEEE Transactions on Smart Grid, vol. 1, no. 1, pp. 11-19, June 2010.

[3] M. Patel (RAPIR Chair), "Real-Time Application of Synchrophasors Improving Reliability," North American Elecricity Reliability Corporation, Princeton, Princeton, NJ, Tech. Rep., 2010, available on-line: http://www.naspi.org/news/rapirfinaldraft20101017.pdf.

[4] M. Zima, M. Larsson, P. Korba, C. Rehtanz, and G. Andersson, "Design Aspects for Wide-Area Monitoring and Control Systems," Proceedings of the IEEE, vol. 93, no. 5, pp. 980-996, May 2005.

[5] J. Patterson, "The Smart Grid: Hadoop at the Tennessee Valley Authority (TVA)," June 2009, available on-line:

http://www.cloudera.com/blog/2009/06/smart-grid-hadooptennesse-valley-authority-tva/.

[6] M. Parashar and J. Mo, "Real Time Dynamics Monitoring System (RTDMS): Phasor Applications for the Control Room," in 42nd Hawaii International Conference on System Sciences, 2009 (HICSS '09), Jan 2009, pp. 1-11.

[7] S. Chakrabarti, E. Kyriakides, B. T. B, C. Deyu, and V. Terzija, "Measurements get together," IEEE Power and Energy Magazine, vol. 7, no. 1, pp. 41-49, January-February 2009.

[8] US Department of Energy, "Smart Grid Investment Grant Selectee Information," 2009, available on-line: http://www.oe.energy.gov/recovery/1264.htm.

[9] C. Martinez, M. Parashar, J. Dyer, and J. Coroas, "Phasor Data Requirements for Real Time Wide-Area Monitoring, Control and Protection Applications," CERTS/EPG, EIPP-Real Time Task Team, Tech. Rep., 2005.

[10] Y. Zhang, P. Markham, T. Xia, L. Chen, Y. Ye, Z. Wu, Z. Yuan, L. Wang, J. Bank, J. Burgett, R. Conners, and Y. Liu, "Wide-Area Frequency Monitoring Network (FNET) Architecture and Applications," IEEE Transactions on Smart Grid, vol. 1, no. 2, pp. 159-167, Sept 2010. 
[11] "F-NET Server Web Display," available on-line: http://fnetpublic.utk.edu/.

[12] “openPDC: The Open Source Phasor Data Concentrator," available online: http://openpdc.codeplex.com/.

[13] J. Zuo, R. Carroll, P. Trachian, J. Dong, S. Affare, B. Rogers, L. Beard, and Y. Liu, "Development of TVA SuperPDC: Phasor applications, tools, and event replay," in IEEE Power and Energy Society General Meeting, 2008.

[14] "openPDC Adapter String Connection Syntax," available on-line: http://openpdc.codeplex.com/.

[15] "The Open Source Time Series Data Historian," available on-line: http://openhistorian.codeplex.com/.

[16] A. Armenia and J. H. Chow, "A Flexible Phasor Data Concentrator Design Leveraging Existing Software Technologies," IEEE Transactions on Smart Grid, vol. 1, no. 1, pp. 73-81, June 2010.

[17] "The TVA Code Library," available on-line: http://tvacodelibrary.codeplex.com/.

[18] L. Vanfretti, L. Dosiek, J. W. Pierre, D. Trudnowski, J. H. Chow, R. García-Valle, and U. Aliyu, "Application of Ambient Analysis Techniques for the Estimation of Electromechanical Oscillations from Measured PMU Data in Four Different Power Systems," European Transactions on Electrical Power, 2010, Special Issue on PMU Data and their Applications, doi: 10.1002/etep.507.

[19] "MATLAB Builder NE for Microsoft .NET framework," available online: http://www.mathworks.com/products/netbuilder/.

[20] "Standard PRC-002-1 - Define Regional Disturbance Monitoring and Reporting Requirements," North American Electric Reliability Corporation (NERC), Princeton, NJ, Tech. Rep., August 2006.

[21] J. Hauer, D. Trudnowski, and J. DeSteese, "A Perspective on WAMS Analysis Tools for Tracking of Oscillatory Dynamics," in IEEE Power Engineering Society General Meeting, 2007, pp. 1-10.

[22] J. Hauer, N. Bhatt, K. Shah, and S. Kolluri, "Performance of "WAMS East" in providing dynamic information for the North East blackout of August 14, 2003," in IEEE Power Engineering Society General Meeting, 2004, pp. $1685-1690$

[23] E. Allen, "Use of COMTRADE for Exchange of PMU Data," in NASPI Workgroup Meeting, Vancouver, British Columbia, June 2010, available on-line: http://www.naspi.org/meetings/workgroup/2010_june/presentations/ session_01/allen_nerc_use_of_comtrade_20100608.pdf.

[24] M. Kezunovic, "Intelligent Design: Substation Data Integration for Enhanced Asset Management Opportunities," IEEE Power and Energy Magazine, vol. 6, no. 6, pp. 37-44, Nov.-Dec. 2010.

[25] "IEEE standard Common Format for Transient Data Exchange (COMTRADE) for power systems," IEEE Std C37.111-1999, Tech. Rep., 1999

[26] R. Almgren, "MySQL Client for MATLAB," available on-line: http://www.courant.nyu.edu/ almgren/mysq1/.

[27] D. Trudnowski, J. Pierre, N. Zhou, J. Hauer, and M. Parashar, "Performance of Three Mode-Meter Block-Processing Algorithms for Automated Dynamic Stability Assessment," IEEE Transactions on Power Systems, vol. 23, no. 2, pp. 680-690, May 2008.

[28] D. Trudnowski and J.W. Pierre, in Inter-area Oscillations in Power Systems: A Nonlinear and Nonstationary Perspective, ser. Power Electronics and Power Systems, A. R. Messina, Ed. Springer, 2009, ch. Signal Processing Methods for Estimating Small-Signal Dynamic Properties from Measured Responses, pp. 1-36.

Moustafa Chenine (Student Member '07) received his B.Sc. with a major in Computer Information Systems from The American College of Greece in 2004. He then joined KTH - The Royal Institute of Technology where he worked towards his M.Sc. (2006) in Information Systems and Licentiate of Engineering in Electrical Engineering (2009). He is a $\mathrm{PhD}$ Student at the department of Industrial Information and Control Systems at KTH, doing research in the field of ICT Infrastructure optimizations for Smart Grid systems, specifically focusing on interoperability, security and performance aspects of Wide Area Monitoring and Control Systems.
Luigi Vanfretti (Student Member '03, M'10) is an Assistant Professor at the Electric Power Systems Division, School of Electrical Engineering, KTH Royal Institute of Technology, Stockholm, Sweden, where he was a PostDoctoral Research Fellow during May 2010.

He obtained the Electrical Engineering Degree (with a Power Engineering concentration) from Universidad de San Carlos de Guatemala in June 2005, and received the Francisco Vela award for outstanding academic achievement and best undergraduate thesis. During Fall 2005, he was a visiting researcher at the Department of Electronics and Electrical Engineering of The University of Glasgow, Scotland.

$\mathrm{He}$ received his MS in 2007 and $\mathrm{PhD}$ in 2009, both in Electric Power Engineering, from the Electrical, Computer, and Systems Engineering (ECSE) Department at Rensselaer Polytechnic Institute (RPI). During Spring 2010 he was a Post-Doctoral Research Associate at RPI working on synchrophasor applications. He was awarded the Charles M. Close Doctoral Prize from RPI in May, 2010 for his research and teaching work at ECSE.

His research interests are modeling, dynamics, stability and control of power systems; applications of PMU data for Smart Transmission Grids, and open source software for power system engineering education.

Sebastian Bengtsson began his studies at KTH, The Royal Institute of Technology, in 2004. He is working towards an M.Sc in Electrical Engineering with main focus on electrical power systems and control theory. He is currently writing his master thesis on power system mode estimation from PMU data at the Electric Power Systems (EPS) department, KTH.

Lars Nordström (M'06) received the M.Sc. degree in electrical engineering and the $\mathrm{Ph} . \mathrm{D}$. degree in industrial control systems, both from KTH-The Royal Institute of Technology, Stockholm, Sweden. He is an Associate Professor in Power System Management and Director of the Swedish Centre of Excellence in Electric Power Engineering, an industry-university research center, located at KTH. His area of research is power systems management and related information exchange, including application of decision theory on information system architectures and the application of ICT to power system problems. Dr. Nordström is chairman of IEC TC57's Swedish committee, and a member of the IEC's Strategic group on Smart grids as well as National Cigre SC D2 representative for Sweden. 